\title{
Effect of sucrose on crying in response to heel stab
}

\author{
J Alison Rushforth, Malcolm I Levene
}

\begin{abstract}
It has been suggested that sucrose acts as an analgesic in the neonatal period. To evaluate this further, 52 infants received either $2 \mathrm{ml}$ of $7 \cdot 5 \%$ sucrose or $2 \mathrm{ml}$ of sterile water before heel stab blood sampling. The duration of crying in response to sampling did not differ in the two groups but was related to level of arousal at the time of stimulation.

(Arch Dis Child 1993; 69: 388-389)
\end{abstract}

In the first few days of life infants may encounter a number of painful stimuli, for example heel stab blood tests. One way in which infants respond to these stimuli is by crying.

Increased awareness of the perception of pain by newborn infants has led to the examination of methods to reduce distress. In 1990 Smith and colleagues produced a reduction in crying of term infants by the administration of oral sucrose solution. ${ }^{1}$ Blass and Hoffmeyer were able to show that oral sucrose solution given to infants resulted in a shorter duration of crying when compared with infants given water. ${ }^{2}$ On the basis of these results they concluded that sucrose acted as an analgesic. The mechanism of this analgesic action has not been identified in human newborns. However, in rat pups, the positive calming influence and apparent analgesic powers of oral sucrose solution are negated by pretreatment with naltrexone, an opiate antagonist, suggesting a possible role of the endogenous opiate system. ${ }^{3}$ We studied the effects of sucrose in a group of term neonates undergoing routine heel stab blood tests.

Academic Unit of Paediatrics and Child Health, Clarendon Wing, The General Infirmary at Leeds, Belmont Grove, Leeds LS2 2NS

J Alison Rushforth

Malcolm I Levene

Correspondence to: Dr Rushforth.

Accepted 17 May 1993

Characteristics of the two groups; numbers are median (range)

\begin{tabular}{lcc}
\hline & Water $(n=26)$ & Sucrose $(n=26)$ \\
\hline Male sex & 10 & 10 \\
Gestational age (weeks) & $39(37-42)$ & $38(37-40)$ \\
Birth weight (g) & $3210(1960-4400)$ & $3000(1840-4350)$ \\
Age (days) & $6(2-6)$ & $6(2-7)$ \\
Time since last feed (min) & $180(60-280)$ & $180(45-300)$ \\
Arousal score at outset & $2(0-4)$ & $2(0-4)$ \\
Method of delivery & 9 & 8 \\
$\quad$ Vaginal & 2 & 1 \\
Forceps & 15 & 17 \\
Caesarean section & &
\end{tabular}
Healthy full term infants were randomly allocated to receive an equal quantity of either $\mathbf{7 . 5 \%}$ sucrose solution or sterile water before routine heel stab blood tests.

All blood samples were taken by a single investigator using an Autolet 2000 Superlite. Infants were taken to a warm, quiet nursery for blood sampling and were fully clothed apart from the foot which was to be used for sampling. Parents were present if they wished, but did not speak to or touch the baby during the procedure. The $2 \mathrm{ml}$ of test solution was administered by dropper into the mouth over a one minute period. The infants were then observed for a further minute to assess behavioural state before sampling; these were as outlined by Gladman and Chiswick and are as follows. ${ }^{4}$ 0: Sleeping, no movement, regular breathing. 1: Sleeping, small movements, irregular breathing. 2: Quiet awake, slight movement. 3: Active awake, moderate movement. 4: Crying and vigorous movement.

Crying during sampling and the three minutes after sampling (recovery phase) was recorded on a standard audio tape recorder and analysed blindly at a later date for the duration of crying.

The study was approved by the hospital ethics committee and informed parental consent was obtained before the study in each case.

\section{Results}

Fifty two newborn infants were recruited. The sucrose and water groups were comparable with respect to sex, gestational age, birth weight, postnatal age, mode of delivery, and time since last feed (table). All infants had a blood glucose concentration (measured by reagent strip) of between 4 and $7 \mathrm{mmol} / 1$.

There was no difference in the median percentage time spent crying in each group during sampling $(74.3 \%$ in the sucrose group, $73.2 \%$ in the water group) and, using the Mann-Whitney test, no difference in the duration of cry in the first $(p=0.65)$, second $(p=0.52)$, and third $(p=0.72)$ minutes of the recovery period. Neither was there a difference in the time to cessation of crying $(p=0 \cdot 16)$.

There was, however, a correlation between arousal scores immediately before blood sampling and the total time spent crying in the recovery phase. Infants who had a higher arousal score before sampling showed a longer duration of crying in the recovery period $(r=0.601 ; \mathrm{p}=0.002$ for sucrose group and $r=0.708 ; \mathrm{p}=0.002$ for water group).

\section{Discussion}

We were unable to duplicate the results of Blass et al in this population of infants ${ }^{1-3}$; there was no evidence to suggest that sucrose reduced infant crying in response to painful heel stab. It seems unlikely that this lack of response could be attributed to our use of a weaker sucrose solution $(7.5 \% \mathrm{w} / \mathrm{v}$ rather than the $12 \% \mathrm{w} / \mathrm{v}$ used by Blass $e t a l$ ), as calming effects have been shown following oral administration of very small volumes of $7 \cdot 5 \%$ sucrose solution to both human newborns $^{1}$ and rat pups. ${ }^{3}$ 
As expected there was a correlation between arousal at the onset of sampling and total duration of crying. It is often noted by technicians taking regular samples from infants that those who are still or asleep often cry less in response to sampling than infants who are active or 'fussing'.
1 Smith BA, Fillion TJ, Blass EM. Orally mediated sources of calming in 1 to 3 day old human infants. Developmental Psychology 1990; 26: 731-7.

2 Blass EM, Hoffmeyer LB. Sucrose as an analgesic for newborn infants. Pediatrics 1991; 87: 215-8.

3 Blass EM, Fitzgerald E, Kehoe P. Interactions between sucrose, pain and isolation distress. Pharmacology sucrose, pain and isolation distress.
Biochemistry and Behaviour 1987; 26: 483-9.

4 Gladman G, Chiswick ML. Skin conductance and arousal in the newborn. Arch Dis Child 1990; 65: 1063-6.

\section{Neonatal cerebral venous thrombosis}

Have you ever wondered what it is that makes people send their papers to one journal rather than another? Presumably a major factor is their judgment as regards likely success, but surely the predicted readership is important. Take Annals of Neurology, for instance. Now, I would guess that the average reader of that splendid journal would be hard pressed to choose between a paper on neonatal neurology and the latest revision of the Ulan Bator and district public bus timetable as regards relevance to his practice. Similarly, most neonatologists will consult the journal at a frequency approximating to that with which an Englishman wins the Wimbledon singles championship. Nevertheless, the July 1992 issue contains a paper of considerable relevance to paediatricians.

Reports of neonatal cerebral venous thrombosis involving either the dural sinuses or deeper veins are uncommon and have usually described a very small number of babies with thrombosis either idiopathic or secondary to conditions such as severe birth asphyxia, polycythaemia, dehydration, sepsis, or protein C deficiency. A report in 1988 described 17 neonates with dural sinus thrombosis detected on computed tomography over a period of eight years. ${ }^{1}$ Now workers in Boston have used both computed tomography and magnetic resonance imaging to show cerebral venous thrombosis in seven newborn infants seen at the Floating Hospital for Infants and Children over a period of only three months (Michael J Rivkin and colleagues, Annals of Neurology 1992; 32: 51-6).

Six of the seven were born at term and the other at 32 weeks' gestation. Three presented with a single focal seizure and four with lethargy. Computed tomography showed evidence of dural sinus thrombosis in six babies and of frontal lobe hypoplasia in the seventh. Magnetic resonance imaging identified venous thrombosis in all seven and in four babies it showed more extensive venous thrombosis than had been seen on the computed tomogram. In addition to standard magnetic resonance images, phase imaging was used and this technique, which is able to demonstrate blood flow in cerebral veins, showed obstruction to flow in all seven patients.

The condition appears to be relatively benign as none of the babies had recurrent seizures and the only one to be neurologically abnormal at follow up was the baby with hypoplastic frontal lobes. The thrombosis was designated idiopathic in all seven and no baby was given anticoagulant or thrombolytic treatment. It seems possible that the condition is more common than we realise. 